\title{
MOTIONS ON CURVES AND SURFACES USING GEOMETRIC
} ALGEBRA

\author{
Selahattin ASLAN and Yusuf YAYLI \\ Department of Mathematics, Ankara University, Ankara, TURKEY
}

\begin{abstract}
Geometric algebra is a useful tool to overcome some problems in kinematics. Thus, the geometric algebra has attracted the attention of many researchers. In this paper, quaternion operators on curves and surfaces in Euclidean 3-space are defined by using geometric algebra. These operators generate the curves or the surfaces from the points, curves or surfaces. Using quaternion operators, we obtain motions that have orbits along the generated curve or surface. Also, these motions are expressed as 1-parameter or 2-parameter homothetic motions.
\end{abstract}

\section{INTRODUCTION}

Kinematics is a research field of geometry to describe the motion of points, lines and other geometric objects. Thus, kinematics is used in many fields such as physics, mechanics, robotics and neuroscience. Homothetic motion is one of the most commonly researched topic in kinematics. 1-parameter and 2-parameter homothetic motions were researched in Euclidean 3-space $\mathbb{E}^{3}[1,2]$. Yayl gave homothetic motions in Euclidean 4-space with Hamilton operators [3].

Sir William Rowan Hamilton [4] interpreted the quaternions as an extension to the complex numbers in 1843 . K. Shoemake defined the system of rotation in $\mathbb{E}^{3}$ by using quaternions [5]. Quaternions are more useful than Euler angles and matrices in representing of rotations of vectors. Therefore, quaternions have been used in many fields such as computer graphics, robotics and control theory.

Some problems and difficulties have been encountered in modeling of the mathematics of 3-dimensional (3D) kinematics. These difficulties have been tried to overcome by using quaternions. Bayro-Corrochano [6] used geometric algebra for the mathematical model of 3D kinematics of eye movements. Then, Leclercq at

2020 Mathematics Subject Classification. 11R52, 53A04, 53A05.

Keywords. Geometric algebra, curves, surfaces, quaternions, rotation matrices, homothetic motions.

@ selahattinnaslan@gmail.com-Corresponding author; yayli@science.ankara.edu.tr

(D) 0000-0001-5322-3265; 0000-0003-4398-3855.

(C)2022 Ankara University Communications Faculty of Sciences University of Ankara Series A1 Mathematics and Statistics 
al. modeled some movements in 3D kinematics such as rotations, translations and screw movements [7]. In [8], an isomorphism was given between the algebra of split semi-quaternions and the Clifford algebra $C l_{1,0,1}$. Moreover, semi-Euclidean planar motion was defined by using the algebra of split semi-quaternions.

Some surfaces were obtained by quaternions or homothetic motions in [9-15]. Some results have been achieved about these surfaces using quaternions. Also, using quaternions in the shape operator expressed by Darboux frame, we defined the quaternionic shape operator [16]. Moreover, we used the quaternionic shape operator in researching of the differential properties of surfaces.

In this study, we define quaternion operators using curves and surfaces in $\mathbb{E}^{3}$. These operators have allowed us to obtain a quaternionic or a homothetic motion on each curve and surface in $\mathbb{E}^{3}$. These motions have orbits along curves or surfaces. Quaternion operator with curve orbit converts a point to a curve or a curve to a curve. This operator is expressed as 1-parameter homothetic motion. Similarly, quaternion operator with surface orbit converts a point to a surface, a curve to a surface, or a surface to a surface. Moreover, quaternion operator with surface orbit is expressed as 2-parameter homothetic motion. Finally, we give some applications of the quaternion operators.

\section{Preliminaries}

In this section, definitions and some algebraic properties of the concepts real quaternions, homothetic motions and geometric algebra will be given to provide a background.

The set $H=\left\{q=a_{0}+a_{1} i+a_{2} j+a_{3} k: a_{0}, a_{1}, a_{2}, a_{3} \in R\right\}$ of real quaternions is equal to the 4 -dimensional vector space $R^{4}$. Quaternions have a basis $\{1, i, j, k\}$ shortly given with some properties as

$$
i^{2}=j^{2}=k^{2}=i j k=-1 .
$$

The set of real quaternion is associative and not commutative algebra. 1 is identity element of $H$. Scalar and vector component of $q$ are $S(q)=a_{0} \in R$ and $V(q)=$ $a_{1} i+a_{2} j+a_{3} k \in \mathbb{E}^{3}$, respectively. We can write quaternion $q$ as $q=S(q)+V(q)$. If $S(q)=0, q$ is called pure quaternion. Quaternion product $*$ of $q=S(q)+V(q)$ and $p=S(p)+V(p)$ is defined as

$$
q * p=S(q) S(p)-V(q) \cdot V(p)+S(q) V(p)+S(p) V(q)+V(q) \times V(p) .
$$

Conjugate, norm, modulus and inverse of $q$ is

$$
\begin{gathered}
\bar{q}=a_{0}-a_{1} i-a_{2} j-a_{3} k, \\
N_{q}=\bar{q} * q=a_{0}^{2}+a_{1}^{2}+a_{2}^{2}+a_{3}^{2}, \\
|q|=\sqrt{N_{q}}, \\
q^{-1}=\frac{\bar{q}}{N_{q}}, \quad N_{q} \neq 0,
\end{gathered}
$$


respectively. If $N_{q}=1, q$ is called unit quaternion. A unit quaternions can be written in the trigonometric form as $q=\cos \theta+\sin \theta \boldsymbol{v}$, where $\boldsymbol{v} \in \mathbb{E}^{3}$ and $\|\boldsymbol{v}\|=1$. Let $\boldsymbol{v}_{1}$ and $\boldsymbol{v}_{2}$ be unit vectors in $\mathbb{E}^{3}$ (i.e., pure quaternions), and $\theta=\arccos \left(\boldsymbol{v}_{1} \cdot \boldsymbol{v}_{2}\right)$, Thus, the unit quaternion $q$ can be given as

$$
q=\boldsymbol{v}_{2} * \boldsymbol{v}_{1}^{-1}=\cos \theta+\sin \theta \boldsymbol{v},
$$

where $\boldsymbol{v}=\frac{\boldsymbol{v}_{1} \times \boldsymbol{v}_{2}}{\left\|\boldsymbol{v}_{1} \times \boldsymbol{v}_{2}\right\|}$. \|\| is the modulus in $\mathbb{E}^{3}$. Unit quaternion $q=\cos \theta+\sin \theta \boldsymbol{v}$ rotates the vector $\boldsymbol{v}_{1}$ to the vector $\boldsymbol{v}_{2}$ around the axis vector $\boldsymbol{v}$, see Figure 1. For further information about real quaternions, see [3-5, 17].

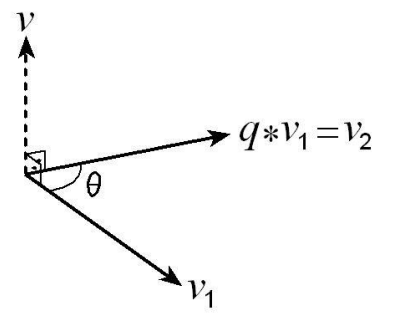

FiguRE 1. Rotation with unit quaternion

Let $p=a_{0}+a_{1} i+a_{2} j+a_{3} k$ be a unit quaternion and $w$ be a pure quaternion (i.e., vector in $\mathbb{E}^{3}$ ). Linear mapping $\phi$ can be defined as

$$
\phi: \mathbb{E}^{3} \rightarrow \mathbb{E}^{3}, \quad \phi(\boldsymbol{w})=p * \boldsymbol{w} * p^{-1} .
$$

Matrix corresponding to the linear mapping $\phi$ can be given as

$$
R=\left[\begin{array}{ccc}
a_{0}^{2}+a_{1}^{2}-a_{2}^{2}-a_{3}^{2} & -2 a_{0} a_{3}+2 a_{1} a_{2} & 2 a_{0} a_{2}+2 a_{1} a_{3} \\
2 a_{0} a_{3}+2 a_{1} a_{2} & a_{0}^{2}+a_{2}^{2}-a_{1}^{2}-a_{3}^{2} & 2 a_{2} a_{3}-2 a_{0} a_{1} \\
2 a_{1} a_{3}-2 a_{0} a_{2} & 2 a_{0} a_{1}+2 a_{2} a_{3} & a_{0}^{2}+a_{3}^{2}-a_{2}^{2}-a_{1}^{2}
\end{array}\right],
$$

where $R$ is orthogonal since $R R^{T}=I$ and $\operatorname{det} R=1$. Thus, $\phi$ represents a rotation in $\mathbb{E}^{3}$. If unit quaternion $p$ is in the form

$$
p=\cos \theta+\sin \theta \boldsymbol{v},
$$

then $\phi(\boldsymbol{w})$ rotates the vector $\boldsymbol{w}$ by $2 \theta[5]$.

1-parameter homothetic motion in $\mathbb{E}^{3}$ can be given as

$$
y(t)=h(t) A(t) \boldsymbol{x}(t)+\boldsymbol{c}(t),
$$

where $\boldsymbol{y}$ and $\boldsymbol{x}$ are the position vectors of the same point in the fixed space $R^{\imath}$ and the moving space $R$, respectively. $h, A$ and $c$ are homothetic scalar, orthogonal matrix and translation vector, respectively. And " $t$ " is homothetic parameter $[1$, $2]$. 
Similarly, 2-parameter homothetic motion in $\mathbb{E}^{3}$ can be given as

$$
y(t, s)=h(t, s) A(t, s) \boldsymbol{x}(t, s)+\boldsymbol{c}(t, s),
$$

where $\boldsymbol{y}$ and $\boldsymbol{x}$ are the position vectors of the same point in the fixed space $R^{\imath}$ and the moving space $R$, respectively. $h, A$ and $c$ are homothetic scalar, orthogonal matrix and translation vector, respectively. And " $t$ and $s$ " are homothetic parameters $[1,2]$.

The geometric product of two unit vectors $\boldsymbol{a}$ and $\boldsymbol{b}$ is written as $\boldsymbol{a} * \boldsymbol{b}$ and can be expressed as a sum of its symmetric and antisymmetric parts

$$
\boldsymbol{a} * \boldsymbol{b}=\boldsymbol{a} \cdot \boldsymbol{b}+\boldsymbol{a} \times \boldsymbol{b},
$$

where the inner product $\boldsymbol{a} \cdot \boldsymbol{b}$ and the outer product $\boldsymbol{a} \times \boldsymbol{b}$ are defined by

$$
\begin{aligned}
\boldsymbol{a} \cdot \boldsymbol{b} & =\frac{1}{2}(\boldsymbol{a} * \boldsymbol{b}+\boldsymbol{b} * \boldsymbol{a}), \\
\boldsymbol{a} \times \boldsymbol{b} & =\frac{1}{2}(\boldsymbol{a} * \boldsymbol{b}-\boldsymbol{b} * \boldsymbol{a}) .
\end{aligned}
$$

The inner product of two vectors is the standard scalar or dot product which results in a scalar. The outer or wedge product of two vectors is a new quantity we call a bivector. We think of a bivector as a directed area in the plane containing $\boldsymbol{a}$ and $\boldsymbol{b}$, formed by sweeping $\boldsymbol{a}$ along $\boldsymbol{b}[6]$.

\section{Quaternion Operators}

In this part, we have defined quaternion operators by geometric algebra. By using this operator, we have obtained some results on the curves and surfaces.

Definition 1. Let $\boldsymbol{a}$ and $\boldsymbol{b}$ be vectors in $\mathbb{E}^{3}$. By using the inner product $\boldsymbol{a} \cdot \boldsymbol{b}$ and the vectorial product $\boldsymbol{a} \times \boldsymbol{b}$, quaternion operator can be defined as

$$
Q=\frac{1}{\|\boldsymbol{a}\|^{2}}(\boldsymbol{a} \cdot \boldsymbol{b}+\boldsymbol{a} \times \boldsymbol{b}) .
$$

The quaternion operator $Q$ converts the vector $\boldsymbol{a}$ to the vector $\boldsymbol{b}$ around the axis vector $\boldsymbol{a} \times \boldsymbol{b}$ in the plane formed by $\boldsymbol{a}$ and $\boldsymbol{b}$ as

$$
\begin{aligned}
Q * \boldsymbol{a} & =\frac{1}{\|\boldsymbol{a}\|^{2}}(\boldsymbol{a} \cdot \boldsymbol{b}+\boldsymbol{a} \times \boldsymbol{b}) * \boldsymbol{a} \\
& =\frac{1}{\|\boldsymbol{a}\|^{2}}(-(\boldsymbol{a} \times \boldsymbol{b}) \cdot \boldsymbol{a}+(\boldsymbol{a} \cdot \boldsymbol{b}) \boldsymbol{a}+(\boldsymbol{a} \times \boldsymbol{b}) \times \boldsymbol{a}) \\
& =\frac{1}{\|\boldsymbol{a}\|^{2}}((\boldsymbol{a} \cdot \boldsymbol{b}) \boldsymbol{a}+(\boldsymbol{a} \cdot \boldsymbol{a}) \boldsymbol{b}-(\boldsymbol{b} \cdot \boldsymbol{a}) \boldsymbol{a}) \\
& =\frac{1}{\|\boldsymbol{a}\|^{2}}\|\boldsymbol{a}\|^{2} \boldsymbol{b}
\end{aligned}
$$




$$
=\boldsymbol{b}
$$

where $\boldsymbol{a}$ and $\boldsymbol{b}$ are pure quaternion. Using $\boldsymbol{a} \cdot \boldsymbol{b}=\|\boldsymbol{a}\|\|\boldsymbol{b}\| \cos \theta$ and $\|\boldsymbol{a} \times \boldsymbol{b}\|=$ $\|\boldsymbol{a}\|\|\boldsymbol{b}\| \sin \theta$ in Eq. (10), we get

$$
\begin{aligned}
Q & =\frac{1}{\|\boldsymbol{a}\|^{2}}(\boldsymbol{a} \cdot \boldsymbol{b}+\boldsymbol{a} \times \boldsymbol{b}) \\
& =\frac{1}{\|\boldsymbol{a}\|^{2}}\left(\|\boldsymbol{a}\|\|\boldsymbol{b}\| \cos \theta+\|\boldsymbol{a}\|\|\boldsymbol{b}\| \sin \theta \frac{\boldsymbol{a} \times \boldsymbol{b}}{\|\boldsymbol{a} \times \boldsymbol{b}\|}\right) \\
& =\frac{\|\boldsymbol{b}\|}{\|\boldsymbol{a}\|}(\cos \theta+\sin \theta \boldsymbol{v}) \\
& =h q .
\end{aligned}
$$

where $q=\cos \theta+\sin \theta \boldsymbol{v}, h=\frac{\|\boldsymbol{b}\|}{\|\boldsymbol{a}\|}$ and $\boldsymbol{v}=\frac{\boldsymbol{a} \times \boldsymbol{b}}{\|\boldsymbol{a} \times \boldsymbol{b}\|}$. Thus, quaternion operator $Q$ can be given as $Q=h q$.

Hence Eq. (11) can be expressed as

$$
Q * \boldsymbol{a}=h q * \boldsymbol{a} .
$$

$Q * \boldsymbol{a}=h q * \boldsymbol{a}$ can be given in Figure 2.

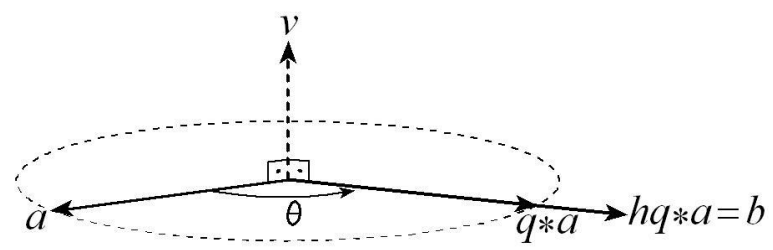

FiguRE 2. Quaternion operator

\subsection{Quaternion Operator with Curve Orbit.}

Theorem 1. Let $\alpha(t)$ and $P$ be a curve and a point in $\mathbb{E}^{3}$, respectively. Quaternion operator can be given as

$$
Q(t)=\frac{1}{\|P\|^{2}}(P \cdot \alpha(t)+P \times \alpha(t)) .
$$

$Q(t)$ generates the curve $\alpha(t)$ from the point $P$ as

$$
Q(t) * P=\alpha(t),
$$

where $\alpha(t)$ is the orbit of $Q(t) * P$ and $P, \alpha(t)$ are pure quaternions. 
Proof. The quaternion product of quaternion operator $Q(t)$ and the pure quaternion $P$ can be given as

$$
\begin{aligned}
Q(t) * P & =\frac{1}{\|P\|^{2}}(P \cdot \alpha(t)+P \times \alpha(t)) * P \\
& =\frac{1}{\|P\|^{2}}((P \cdot \alpha(t)) P+(P \times \alpha(t)) \times P) \\
& =\frac{1}{\|P\|^{2}}(P \cdot P) \alpha(t) \\
& =\frac{1}{\|P\|^{2}}\|P\|^{2} \alpha(t) \\
& =\alpha(t) .
\end{aligned}
$$

Quaternion operator $Q(t)$ generates the curve $\alpha(t)$ from the point $P$.

Remark 1. Using $P \cdot \alpha(t)=\|P\|\|\alpha(t)\| \cos \theta(t)$ and $\|P \times \alpha(t)\|=\|P\|\|\alpha(t)\| \sin \theta(t)$, the quaternion operator $Q(t)$ can be given by unit quaternion $q(t)=\cos \theta(t)+$ $\sin \theta(t) \boldsymbol{v}(t)$, where $\boldsymbol{v}(t)=\frac{P \times \alpha(t)}{\|P \times \alpha(t)\|}$ is rotation axis, as

$$
\begin{aligned}
Q(t) & =\frac{1}{\|P\|^{2}}(P \cdot \alpha(t)+P \times \alpha(t)) \\
& =\frac{1}{\|P\|^{2}}\left(P \cdot \alpha(t)+\|P \times \alpha(t)\| \frac{P \times \alpha(t)}{\|P \times \alpha(t)\|}\right) \\
& =\frac{\|\alpha(t)\|}{\|P\|}(\cos \theta(t)+\sin \theta(t) \boldsymbol{v}(t)) \\
& =\frac{\|\alpha(t)\|}{\|P\|} q(t) .
\end{aligned}
$$

Thus, Eq. (14) can be given as

$$
Q(t) * P=\frac{\|\alpha(t)\|}{\|P\|} q(t) * P .
$$

Theorem 2. $Q(t) * P$ given in Eq. (13) can be expressed by 1-parameter homothetic motion in $\mathbb{E}^{3}$ as

$$
Q(t) * P=h(t) R(t) P
$$

where $R(t)$ is the orthogonal matrix satisfying $R(t) P=q(t) * P, q(t)=\frac{Q(t)}{|Q(t)|}$, $h(t)=\frac{\|\alpha(t)\|}{\|P\|}$ is a homothetic scalar and $t$ is homothetic parameter.

Proof. If we take the unit quaternion $p=\cos \theta+\sin \theta \boldsymbol{v}$ in Eq. (3) as $q_{1}(t)=$ $\cos \frac{\theta(t)}{2}+\sin \frac{\theta(t)}{2} \boldsymbol{v}(t)$, we get the orthogonal matrix corresponding to the mapping 
$\phi$ as

$$
R=\left[\begin{array}{ccc}
\left(\cos ^{2} \frac{\theta}{2}+\sin ^{2} \frac{\theta}{2}\left(2 v_{1}^{2}-1\right)\right. & -2 \sin \frac{\theta}{2}\left(\cos \frac{\theta}{2} v_{3}-\sin \frac{\theta}{2} v_{1} v_{2}\right) & 2 \sin \frac{\theta}{2}\left(\cos \frac{\theta}{2} v_{2}+\sin \frac{\theta}{2} v_{1} v_{3}\right) \\
2 \sin \frac{\theta}{2}\left(\cos \frac{\theta}{2} v_{3}+\sin \frac{\theta}{2} v_{1} v_{2}\right) & \cos ^{2} \frac{\theta}{2}+\sin ^{2} \frac{\theta}{2}\left(2 v_{2}^{2}-1\right) & 2 \sin \frac{\theta}{2}\left(\sin \frac{\theta}{2} v_{2} v_{3}-\cos \frac{\theta}{2} v_{1}\right) \\
2 \sin \frac{\theta}{2}\left(\sin \frac{\theta}{2} v_{1} v_{3}-\cos \frac{\theta}{2} v_{2}\right) & 2 \sin \frac{\theta}{2}\left(\cos \frac{\theta}{2} v_{1}+\sin \frac{\theta}{2} v_{2} v_{3}\right) & \cos ^{2} \frac{\theta}{2}+\sin ^{2} \frac{\theta}{2}\left(2 v_{3}^{2}-1\right)
\end{array}\right]
$$

where $\boldsymbol{v}(t)=\left(v_{1}(t), v_{2}(t), v_{3}(t)\right)$. In this case, matrix $R(t)$ performs a rotation by angle $2 \frac{\theta(t)}{2}=\theta(t)$ of the vector $P$ around the axis $\boldsymbol{v}(t)$. Thus, we can give the equalities

$$
q(t) * P=\phi(P)=R(t) P .
$$

Using these equations and $h(t)=\frac{\|\alpha(t)\|}{\|P\|}$, we get

$$
\begin{aligned}
Q(t) * P & =h(t) q(t) * P \\
& =h(t) R(t) P .
\end{aligned}
$$

It means that $Q(t) * P$ can be expressed as 1-parameter homothetic motion $Q(t) *$ $P=h(t) R(t) P$ in $\mathbb{E}^{3}$.

If we take the point $P$ on the curve $\alpha(t)$ as $P=\alpha\left(t_{0}\right)$, then $Q(t) * \alpha\left(t_{0}\right)=$ $h(t) R(t) \alpha\left(t_{0}\right)$ can be given in Figure 3 .

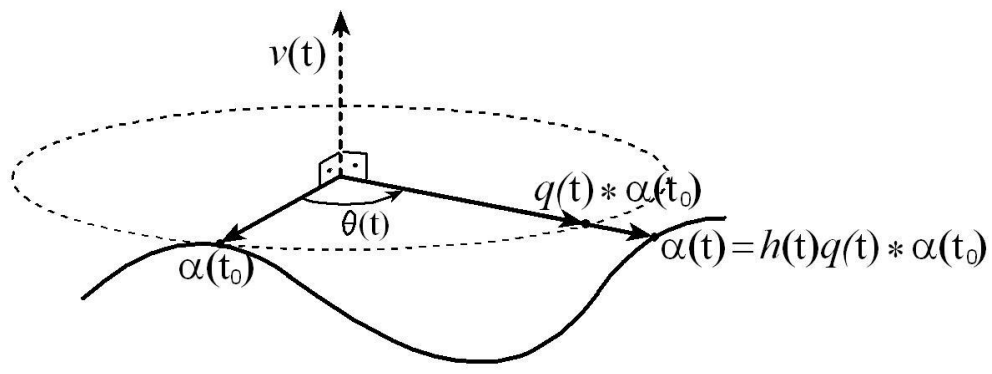

FiguRE 3. Quaternion operator with curve orbit

Corollary 1. If we take the curve $\alpha(t)$ on a surface $M(t, s)$, then orbit of motions obtained in Theorem 1 and Theorem 2 can be confined on $M(t, s)$. Thus, these operators can allow us to obtain a 1-parameter motion on every surface in $\mathbb{E}^{3}$.

Proposition 1. Let $\alpha(t)$ and $\beta(t)$ be curves in $\mathbb{E}^{3}$. Quaternion operator $Q(t)$ can be given as

$$
Q(t)=\frac{1}{\|\alpha(t)\|^{2}}(\alpha(t) \cdot \beta(t)+\alpha(t) \times \beta(t))
$$

where $\alpha(t)$ and $\beta(t)$ are position vectors in $\mathbb{E}^{3}$. This quaternion operator converts the curve $\alpha(t)$ to the curve $\beta(t)$ as 


$$
Q(t) * \alpha(t)=\beta(t)
$$

where the curve $\beta(t)$ is the orbit of $Q(t) * \alpha(t)$. Moreover, $Q(t) * \alpha(t)$ can be given by 1-parameter homothetic motion as

$$
Q(t) * \alpha(t)=h(t) R(t) \alpha(t),
$$

where $R(t)$ is the orthogonal matrix satisfying $R(t) \alpha(t)=q(t) * \alpha(t), q(t)=\frac{Q(t)}{|Q(t)|}$, $h(t)=\frac{\|\beta(t)\|}{\|\alpha(t)\|}$ is a homothetic scalar and $t$ is homothetic parameter.

\subsection{Quaternion Operator with Surface Orbit.}

Theorem 3. Let $M(t, s)$ and $P$ be a surface and a point in $\mathbb{E}^{3}$, respectively. Quaternion operator $Q(t, s)$ can be defined as

$$
Q(t, s)=\frac{1}{\|P\|^{2}}(P \cdot M(t, s)+P \times M(t, s)),
$$

where $M(t, s)$ and $P$ are position vectors in $\mathbb{E}^{3}$. The operator $Q(t, s)$ generates the surface $M(t, s)$ from the point $P$ as

$$
Q(t, s) * P=M(t, s),
$$

where $M(t, s)$ is the orbit of the $Q(t, s) * P$ and $P, M(t, s)$ are pure quaternions.

Proof. The proof of this theorem is similar to the proof of Theorem 1.

Remark 2. By using $P \cdot M(t, s)=\|P\|\|M(t, s)\| \cos \theta(t, s)$ and $\|P \times M(t, s)\|=$ $\|P\|\|M(t, s)\| \sin \theta(t, s)$, the quaternion operator $Q(t, s)$ with unit quaternion $q(t, s)=$ $\cos \theta(t, s)+\sin \theta(t, s) \boldsymbol{v}(t, s)$ can be given as

$$
Q(t, s)=\frac{\|M(t, s)\|}{\|P\|}(\cos \theta(t, s)+\sin \theta(t, s) \boldsymbol{v}(t, s))
$$

where $\boldsymbol{v}(t, s)=\frac{P \times M(t, s)}{\|P \times M(t, s)\|}$. Eq. (23) can be expressed as

$$
Q(t, s) * P=\frac{\|M(t, s)\|}{\|P\|} q(t, s) * P .
$$

Theorem 4. $Q(t, s) * P$ given in Eq. (25) can be given by 2-parameter homothetic motion in $\mathbb{E}^{3}$ as

$$
Q(t, s) * P=h(t, s) R(t, s) P,
$$

where $R(t, s)$ is the orthogonal matrix satisfying $R(t, s) P=q(t, s) * P, q(t, s)=$ $\frac{Q(t, s)}{|Q(t, s)|}, h(t, s)=\frac{\|M(t, s)\|}{\|P\|}$ is a homothetic scalar, and $t, s$ are homothetic parameters.

Proof. The proof of this theorem is similar to the proof of Theorem 2. 
If we take the point $P$ on the surface $M(t, s)$ as $P=M\left(t_{0}, s_{0}\right)$, then $Q(t, s) *$ $M\left(t_{0}, s_{0}\right)=h(t, s) R(t, s) M\left(t_{0}, s_{0}\right)$ can be given in Figure 4 .

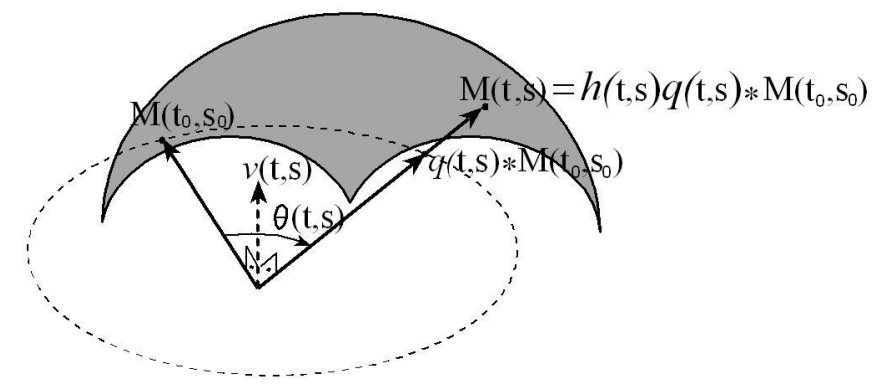

FigURE 4. Quaternion operator with surface orbit

Proposition 2. Let $M(t, s)$ and $\alpha(t)$ be a surface and a curve in $\mathbb{E}^{3}$, respectively. Quaternion operator $Q(t, s)$ can be defined as

$$
Q(t, s)=\frac{1}{\|\alpha(t)\|^{2}}(\alpha(t) \cdot M(t, s)+\alpha(t) \times M(t, s))
$$

where $M(t, s)$ and $\alpha(t)$ are pure quaternions. The operator $Q(t, s)$ generates the surface $M(t, s)$ from the curve $\alpha(t)$ as

$$
Q(t, s) * \alpha(t)=M(t, s)
$$

where $Q(t, s) * \alpha(t)$ has the surface orbit $M(t, s)$.

Corollary 2. $Q(t, s) * \alpha(t)$ given in Eq. (28) can be given by 2-parameter homothetic motion in $\mathbb{E}^{3}$ as

$$
Q(t, s) * \alpha(t)=h(t, s) R(t, s) \alpha(t),
$$

where $R(t, s)$ is the orthogonal matrix satisfying $R(t, s) \alpha(t)=q(t, s) * \alpha(t), q(t, s)=$ $\frac{Q(t, s)}{|Q(t, s)|}, h(t, s)=\frac{\|M(t, s)\|}{\|\alpha(t)\|}$ is a homothetic scalar, and $t, s$ are homothetic parameters.

Proposition 3. Let $M(t, s)$ and $N(t, s)$ be surfaces in $\mathbb{E}^{3}$. Quaternion operator can be defined as

$$
Q(t, s)=\frac{1}{\|M(t, s)\|^{2}}(M(t, s) \cdot N(t, s)+M(t, s) \times N(t, s)),
$$

where $M(t, s)$ and $N(t, s)$ are pure quaternions. The operator $Q(t, s)$ generates the surface $N(t, s)$ from the surface $M(t, s)$ as

$$
Q(t, s) * M(t, s)=N(t, s),
$$

where $Q(t, s) * M(t, s)$ has the surface orbit $N(t, s)$. 
Corollary 3. $Q(t, s) * M(t, s)$ given in Eq. (31) can be given by 2-parameter homothetic motion in $\mathbb{E}^{3}$ as

$$
Q(t, s) * M(t, s)=h(t, s) R(t, s) M(t, s),
$$

where $R(t, s)$ is the orthogonal matrix satisfying $R(t, s) M(t, s)=q(t, s) * M(t, s)$, $q(t, s)=\frac{Q(t, s)}{|Q(t, s)|}, h(t, s)=\frac{\|N(t, s)\|}{\|M(t, s)\|}$ is a homothetic scalar, and $t, s$ are homothetic parameters.

\subsection{Applications of Quaternion Operators.}

Example 1. Let $\alpha(t)=(\cos t, \sin t, 0)$ and $\beta(t)=(\cos t, \sin t, t)$ be curves in $\mathbb{E}^{3}$. The quaternion operator $Q(t)$ can be given as

$$
\begin{aligned}
Q(t) & =\frac{1}{\|\alpha(t)\|^{2}}(\alpha(t) \cdot \beta(t)+\alpha(t) \times \beta(t)) \\
& =1+t(\sin t,-\cos t, 0) .
\end{aligned}
$$

The operator $Q(t)$ converts $\alpha(t)$ to $\beta(t)$ as

$$
\begin{aligned}
Q(t) * \alpha(t) & =(1+t(\sin t,-\cos t, 0)) *(\cos t, \sin t, 0) \\
& =(\cos t, \sin t, 0)+(0,0, t) \\
& =(\cos t, \sin t, t) \\
& =\beta(t) .
\end{aligned}
$$

By using $\|\alpha(t)\|=1,\|\beta(t)\|=\sqrt{1+t^{2}}$ and $v(t)=(\sin t,-\cos t, 0)$, the quaternion operator can be given by unit quaternion $q(t)=\cos \theta(t)+\sin \theta(t) v(t)$ as

$$
\begin{aligned}
Q(t) & =\frac{\|\beta(t)\|}{\|\alpha(t)\|} q(t) \\
& =\sqrt{1+t^{2}}(\cos \theta(t)+\sin \theta(t)(\sin t,-\cos t, 0))
\end{aligned}
$$

where $\theta(t)=\arccos \left(\frac{1}{\sqrt{1+t^{2}}}\right)$. For $p(t)=\cos \frac{\theta(t)}{2}+\sin \frac{\theta(t)}{2} v(t)$, the corresponding matrix $R(t)$ to the linear mapping $\phi$ can be obtained as

$$
R(t)=\left[\begin{array}{ccc}
\cos ^{2} \frac{\theta}{2}-\sin ^{2} \frac{\theta}{2}(\cos 2 t) & -\sin ^{2} \frac{\theta}{2} \sin 2 t & -\sin \theta \cos t \\
-\sin ^{2} \frac{\theta}{2} \sin 2 t & \cos ^{2} \frac{\theta}{2}+\sin ^{2} \frac{\theta}{2} \cos 2 t & -\sin \theta \sin t) \\
\sin \theta \cos t & \sin \theta \sin t & \cos \theta
\end{array}\right] .
$$

Thus, Eq. (34) can be given by the 1-parameter homothetic motion as

$$
Q(t) * \alpha(t)=\sqrt{1+t^{2}} R(t) \alpha(t) .
$$


Example 2. Let $\alpha(t)$ be center curve of the tube surface Tube $(t, \theta)$. The tube surface Tube $(t, \theta)$ can be given by the surface $S(t, \theta)=\cos \theta N(t)+\sin \theta B(t)$ as

$$
\begin{aligned}
\text { Tube }(t, \theta) & =\alpha(t)+r(t)(\cos \theta N(t)+\sin \theta B(t)) \\
& =\alpha(t)+r(t) S(t, \theta) .
\end{aligned}
$$

In the study of canal surfaces with quaternions [9] Corollary 1, the unit quaternion $q(t, \theta)=\cos \theta+\sin \theta T(t)$ generates the surface $S(t, \theta)$ from the normal vector $N(t)$, where $\{T(t), N(t), B(t)\}$ is the Frenet frame of $\alpha(t)$. Using definition of quaternion operator, unit quaternion operator $q(t, \theta)$ can be obtained as

$$
\begin{aligned}
Q(t, \theta) & =\frac{1}{\|N(t)\|^{2}}(N(t) \cdot S(t, \theta)+N(t) \times S(t, \theta)) \\
& =(N(t) \cdot(\cos \theta N(t)+\sin \theta B(t))+N(t) \times(\cos \theta N(t)+\sin \theta B(t))) \\
& =(\cos \theta N(t) \cdot N(t)+\sin \theta N(t) \times B(t)) \\
& =\left(\cos \theta\|N(t)\|^{2}+\sin \theta T(t)\right) \\
& =\cos \theta+\sin \theta T(t) \\
& =q(t, \theta) .
\end{aligned}
$$

where $\|N(t)\|=1$. Thus, quaternion operator $q(t, \theta)$ generates the surface $S(t, \theta)$ from the normal vector $N(t)$ as

$$
\begin{aligned}
q(t, \theta) * N(t) & =(\cos \theta+\sin \theta T(t)) * N(t) \\
& =\cos \theta N(t)+\sin \theta T(t) \times N(t) \\
& =\cos \theta N(t)+\sin \theta B(t) \\
& =S(t, \theta),
\end{aligned}
$$

where $S(t, \theta)$ is the surface orbit of $q(t, \theta) * N(t)$. Thus, tube surface can be given by quaternion product

$$
\operatorname{Tube}(t, \theta)=\alpha(t)+r(t) q(t, \theta) * N(t) .
$$

\section{Conclusions}

In this paper, we define quaternion operators using geometric algebra and classify these operators according to their orbits (i.e., curves or surfaces). Quaternion operator with curve orbit generates a curve from a point or a curve. This operator is given as 1-parameter homothetic motion. Similarly, quaternion operator with surface orbit generates a surface from a point, a curve or a surface. Quaternion operator with surface orbit is also expressed as 2-parameter homothetic motion. Thus, quaternion operators can form a homothetic and a quaternionic motion on every surface and curve in $\mathbb{E}^{3}$. Finally, we give some examples of the quaternion operators. 
Author Contribution Statements The authors contributed equally to this work.

Declaration of Competing Interests The authors have no competing interests to declare.

\section{REFERENCES}

[1] Bottema, O., Roth, B., Theoretical Kinematics, North Holland Publ. Com., 1979.

[2] Karger, A., Novák, J., Space Kinematics and Lie Groups, Breach Science Publishers, S.A. Switzerland, 1985.

[3] Yaylı, Y., Homothetic Motions at $\mathbb{R}^{4}$, Mech. Mach. Theory, 27(3) (1992), 303-305. https://doi.org/10.1016/0094-114X(92)90020-I

[4] Hamilton, W. R., On quaternions; or on a new system of imaginaries in algebra, London, Edinburgh, and Dublin Philosophical Magazine and Journal of Science, 25(3) (1844), 489495.

[5] Shoemake, K., Animating rotation with quaternion curves, in Proceedings of the Proceedings of the 12th Annual Conference on Computer Graphics and Interactive Techniques (SIG-GRAPH '85), vol. 19, ACM, New York, NY, USA, (1985), 245-254. https://doi.org/10.1145/325334.325242

[6] Bayro-Corrochano, E., Modeling the 3D kinematics of the eye in the geometric algebra framework, Pattern Recognition, 36(12) (2003), 2993-3012. https://doi.org/10.1016/S00313203(03)00180-8

[7] Leclercq, G., Lefèvre, P., Blohm, G., 3D kinematics using dual quaternions: theory and applications in neuroscience, Frontiers in Behavioral Neuroscience, (2013), p. 7. https://doi.org/10.3389/fnbeh.2013.00007

[8] Bekar, M., Yayl,, Y., Semi-Euclidean quasi-elliptic planar motion, International Journal of Geometric Methods in Modern Physics, 13(7) (2016), p. 11. https://doi.org/10.1142/S0219887816500894

[9] Aslan, S., Yaylı, Y., Canal surfaces with quaternions, Advanced in Applied Clifford Algebras, 26(1) (2016), 31-38. https://doi.org/10.1007/s00006-015-0602-5

[10] Aslan, S., Yayll, Y., Split quaternions and canal surfaces in Minkowski 3-space, Int. J. Geom., 5(2) (2016), 51-61.

[11] Aslan, S., Yayl, Y., Generalized constant ratio surfaces and quaternions, Kuwait J. Sci., 44(1) (2017), 42-47.

[12] Babaarslan, M., Yayli, Y., A new approach to constant slope surfaces with quaternion, ISRN Geom., Article ID 126358, (2012), p. 8 . https://doi.org/10.5402/2012/126358

[13] Gok, I., Quaternionic Approach of canal surfaces constructed by some new ideas, Advanced in Applied Clifford Algebras, 27(2) (2017), 1175-1190. https://doi.org/10.1007/s00006-016-0703-9

[14] Babaarslan, M. , Yayl,, Y., Split Quaternions and spacelike constant slope surfaces in Minkowski 3-space, International Journal of Geometry, 2(1) (2013), 23-33.

[15] Babaarslan, M. , Yaylı, Y., Split quaternions and time-like constant slope surfaces in Minkowski 3-space, International Journal of Geometry, 8(1) (2019), 57-71.

[16] Aslan, S., Yayll, Y., Quaternionic shape operator, Advanced in Applied Clifford Algebras, 27(4) (2017), 2921-2931. https://doi.org/10.1007/s00006-017-0804-0

[17] Hacisalihoğlu, H. H., Geometry of Motions and Theory of Quaternions, Gazi Unv. Publishing, 1983. 